\title{
Bipartite Powers of k-chordal Graphs
}

\author{
L. Sunil Chandran $\|^{\dagger}$ and Rogers Mathew" \\ Department of Computer Science and Automation, Indian Institute of Science, Bangalore, India.
}

received $4^{\text {th }}$ May 2012, revised $17^{\text {th }}$ April 2013, accepted $14^{\text {th }}$ April 2013.

Let $k$ be an integer and $k \geq 3$. A graph $G$ is $k$-chordal if $G$ does not have an induced cycle of length greater than $k$. From the definition it is clear that 3-chordal graphs are precisely the class of chordal graphs. Duchet proved that, for every positive integer $m$, if $G^{m}$ is chordal then so is $G^{m+2}$. Brandstädt et al. in [Andreas Brandstädt, Van Bang Le, and Thomas Szymczak. Duchet-type theorems for powers of HHD-free graphs. Discrete Mathematics, 177(1-3):9-16, 1997.] showed that if $G^{m}$ is $k$-chordal, then so is $G^{m+2}$.

Powering a bipartite graph does not preserve its bipartitedness. In order to preserve the bipartitedness of a bipartite graph while powering Chandran et al. introduced the notion of bipartite powering. This notion was introduced to aid their study of boxicity of chordal bipartite graphs. The $m$-th bipartite power $G^{[m]}$ of a bipartite graph $G$ is the bipartite graph obtained from $G$ by adding edges $(u, v)$ where $d_{G}(u, v)$ is odd and less than or equal to $m$. Note that $G^{[m]}=G^{[m+1]}$ for each odd $m$.

In this paper we show that, given a bipartite graph $G$, if $G$ is $k$-chordal then so is $G^{[m]}$, where $k, m$ are positive integers with $k \geq 4$.

Keywords: $k$-chordal graph, hole, chordality, graph power, bipartite power.

\section{Introduction}

A hole is a chordless (or an induced) cycle in a graph. The chordality of a graph $G$, denoted by $\mathcal{C}(G)$, is defined to be the size of a largest hole in $G$, if there exists a cycle in $G$. If $G$ is acyclic, then its chordality is taken as 0 . A graph $G$ is $k$-chordal if $\mathcal{C}(G) \leq k$. In other words, a graph is $k$-chordal if it has no holes with more than $k$ vertices in it. Chordal graphs are exactly the class of 3-chordal graphs and chordal bipartite graphs are bipartite, 4 -chordal graphs. $k$-chordal graphs have been studied in the literature in [2], [5], [6], [8], [9] and [16]. For example, Chandran and Ram [5] proved that the number of minimum cuts in a $k$-chordal graph is at most $\frac{(k+1) n}{2}-k$. Spinrad[16] showed that $(k-1)$-chordal graphs can be recognized in $O\left(n^{k-3} M\right)$ time, where $M$ is the time required to multiply two $n$ by $n$ matrices.

Powering and its effects on the chordality of a graph has been a topic of interest. The $m$-th power of a graph $G$, denoted by $G^{m}$, is a graph with vertex set $V\left(G^{m}\right)=V(G)$ and edge set $E\left(G^{m}\right)=\{(u, v) \mid u \neq$ $\left.v, d_{G}(u, v) \leq m\right\}$, where $d_{G}(u, v)$ represents the distance between $u$ and $v$ in $G$. Balakrishnan and

\footnotetext{
${ }^{\dagger}$ Email: sunilecsa.iisc.ernet.in

‡Email: rogers@csa.iisc.ernet.in

1365-8050 @ 2013 Discrete Mathematics and Theoretical Computer Science (DMTCS), Nancy, France
} 
Paulraja [1] proved that odd powers of chordal graphs are chordal. Chang and Nemhauser [7] showed that if $G$ and $G^{2}$ are chordal then so are all powers of $G$. Duchet [10] proved a stronger result which says that if $G^{m}$ is chordal then so is $G^{m+2}$. Brandstädt et al. in [3] showed that if $G^{m}$ is $k$-chordal then so is $G^{m+2}$, where $k \geq 3$ is an integer. Studies on families of graphs that are closed under powering can also be seen in the literature. For instance, it is known that interval graphs, proper interval graphs [14], strongly chordal graphs [13], circular-arc graphs [15][12], cocomparability graphs [11] etc. are closed under taking powers.

Subclasses of bipartite graphs, like chordal bipartite graphs, are not closed under powering since the $m$-th power of a bipartite graph need not be even bipartite. Chandran et al. in [4] introduced the notion of bipartite powering to retain the bipartitedness of a bipartite graph while taking power. The $m$-th bipartite power $G^{[m]}$ of a bipartite graph $G$ is the bipartite graph obtained from $G$ by adding edges $(u, v)$ where $d_{G}(u, v)$ is odd and less than or equal to $m$. Note that $G^{[m]}=G^{[m+1]}$ for each odd $m$. It was shown in [4] that the $m$-th bipartite power of a tree is chordal bipartite. The intention there was to construct chordal bipartite graphs of high boxicity. The fact that the chordal bipartite graph under consideration was obtained as a bipartite power of a tree was crucial for proving that its boxicity was high. Since trees are a subclass of chordal bipartite graphs, a natural question that came up was the following: is it true that the $m$-th bipartite power of every chordal bipartite graph is chordal bipartite? In this paper we answer this question in the affirmative. In fact, we prove a more general result.

\section{Our Result}

Let $m, k$ be positive integers with $k \geq 4$. Let $G$ be a bipartite graph. If $G$ is $k$-chordal, then so is $G^{[m]}$. Note that the special case when $k=4$ gives us the following result: chordal bipartite graphs are closed under bipartite powering.

\section{Graph Preliminaries}

Throughout this paper we consider only finite, simple, undirected graphs. For a graph $G$, we use $V(G)$ to denote the set of vertices of $G$. Let $E(G)$ denote its edge set. For every $x, y \in V(G), d_{G}(x, y)$ represents the distance between $x$ and $y$ in $G$. For every $u \in V(G), N_{G}(u)$ denotes its open neighborhood in $G$, i.e. $N_{G}(u)=\{v \mid(u, v) \in E(G)\}$. A path $P$ on the vertex set $V(P)=\left\{v_{1}, v_{2}, \ldots, v_{n}\right\}$ (where $n \geq 2$ ) has its edge set $E(P)=\left\{\left(v_{i}, v_{i+1}\right) \mid 1 \leq i \leq n-1\right\}$. Such a path is denoted by $v_{1} v_{2} \ldots v_{n}$. If $v_{i}, v_{j} \in V(P), v_{i} P v_{j}$ is the path $v_{i} v_{i+1} \ldots v_{j}$. The length of a path $P$ is the number of edges in it and is denoted by $\|P\|$. A cycle $C$ with vertex set $V(C)=\left\{v_{1}, v_{2}, \ldots, v_{n}\right\}$, and edge set $E(C)=\left\{\left(v_{i}, v_{i+1}\right) \mid 1 \leq i \leq n-1\right\} \cup\left\{\left(v_{n}, v_{1}\right)\right\}$ is denoted as $C=v_{1} v_{2} \ldots v_{n} v_{1}$. We use $\|C\|$ to denote the length of cycle $C$.

\section{Holes in Bipartite Powers}

Let $H$ be a bipartite graph. Let $\mathcal{B}(H)$ be a family of graphs constructed from $H$ in the following manner: $H^{\prime} \in \mathcal{B}(H)$ if corresponding to each vertex $v \in V(H)$ there exists a nonempty bag of vertices, say $B_{v}$, in $H^{\prime}$ such that (a) for every $x \in B_{u}, y \in B_{v},(x, y) \in E\left(H^{\prime}\right)$ if and only if $(u, v) \in E(H)$, and (b) vertices within each bag in $H^{\prime}$ are pairwise non-adjacent. Below we list a few observations about $H$ and every $H^{\prime}$ (, where $H^{\prime} \in \mathcal{B}(H)$ ):

Observation 1. $H^{\prime}$ is bipartite. 
Observation 2. $H$ is an induced subgraph of $H^{\prime}$.

Observation 3. Let $k$ be an integer such that $k \geq 4$. If $H$ is $k$-chordal, then so is $H^{\prime}$.

Proof: Any hole of size greater than 4 in $H^{\prime}$ cannot have more than one vertex from the same bag, say $B_{v}$, as such vertices have the same neighborhood. Hence, the vertices of a hole (of size greater than 4 ) in $H^{\prime}$ belong to different bags and thus there is a corresponding hole of the same size in $H$.

Theorem 4. Let $m, k$ be positive integers with $k \geq 4$. Let $G$ be a bipartite graph. If $G$ is $k$-chordal, then so is $G^{[m]}$.

Proof: We prove this by contradiction. Let $p$ denote the size of a largest induced cycle, say $C=$ $u_{0} u_{1} \ldots u_{p-1} u_{0}$, in $G^{[m]}$. Assume $p>k$. Then, $p \geq 6$ (since $k \geq 4$ and $G^{[m]}$ is bipartite). Between each $u_{i-1}$ and $u_{i}$, where $i \in\{0, \ldots, p-1\}$, there exists a shortest path of length not more than $m$ in $G^{(i)}$. Let $P_{i}$ be one such shortest path between $u_{i-1}$ and $u_{i}$ in $G$.

Let $H$ be the subgraph induced on the vertex set $\bigcup_{i=0}^{p-1} V\left(P_{i}\right)$ in $G$. As mentioned in the beginning of this section, construct a graph $H^{\prime}$ from $H$, where $H^{\prime} \in \mathcal{B}(H)$, in the following manner: for each $v \in V(H)$, let $\left|B_{v}\right|=\left|\left\{P_{i} \mid 0 \leq i \leq p-1, v \in V\left(P_{i}\right)\right\}\right|$ i.e., let $B_{v}$ have as many vertices as the number of paths in $\left\{P_{0} \ldots P_{p-1}\right\}$ that share vertex $v$ in $H$. For each $i \in\{0, \ldots, p-1\}$, let $Q_{i}^{\prime}=u_{i-1} Q_{i}$ be a shortest path between $u_{i-1}$ and $u_{i}$ in $H^{\prime}$ such that no two paths $Q_{i}$ and $Q_{j}$ (where $i \neq j$ ) share a vertex ${ }^{(i)}$ . From our construction of $H^{\prime}$ from $H$ it is easy to see that such paths exist. Let $Q_{i}=v_{i, 1} v_{i, 2} \ldots v_{i, r_{i}} u_{i}$, where $r_{i}=\left\|Q_{i}\right\| \geq 0$. Thus, $Q_{i}^{\prime}=u_{i-1} v_{i, 1} v_{i, 2} \ldots v_{i, r_{i}} u_{i}$. Clearly, $\left\|Q_{i}^{\prime}\right\|=\left\|P_{i}\right\| \leq m$. The reader may also note that the cycle $C\left(=u_{0} u_{1} \ldots u_{p-1} u_{0}\right)$ which is present in $G^{[m]}$ will be present in $H^{[m]}$ and thereby in $H^{\prime[m]}$ too.

In order to prove the theorem, it is enough to show that there exists an induced cycle of size at least $p$ in $H^{\prime}$. Then by combining Observation 3 and the fact that $H$ is an induced subgraph of $G$, we get $k \geq \mathcal{C}(G) \geq \mathcal{C}(H) \geq \mathcal{C}\left(H^{\prime}\right) \geq p$ contradicting our assumption that $p>k$. Hence, in the rest of the proof we show that $\mathcal{C}\left(H^{\prime}\right) \geq p$.

Consider the following drawing of the graph $H^{\prime}$. Arrange the vertices $u_{0}, u_{1}, \ldots, u_{p-1}$ in that order on a circle in clockwise order. Between each $u_{i-1}$ and $u_{i}$ on the circle arrange the vertices $v_{i, 1}, v_{i, 2}, \ldots, v_{i, r_{i}}$ in that order in clockwise order. Recall that these vertices are the internal vertices of path $Q_{i}^{\prime}$.

Claim 4.1. In this circular arrangement of vertices of $H^{\prime}$, each vertex has an edge (in $H^{\prime}$ ) with both its left neighbor and right neighbor in the arrangement.

Let $x_{1}, x_{2} \in V\left(H^{\prime}\right)$, where $x_{1} \in V\left(Q_{i}\right), x_{2} \in V\left(Q_{j}\right)$. We define the clockwise distance from $x_{1}$ to $x_{2}$, denoted by clock_dist $\left(x_{1}, x_{2}\right)$, as the minimum non-negative integer $s$ such that $j=i+s$. Similarly, the clockwise distance from $x_{2}$ to $x_{1}$, denoted by clock_dist $\left(x_{2}, x_{1}\right)$, is the minimum non-negative integer $s^{\prime}$ such that $i=j+s^{\prime}$. Let $x, y, z \in V\left(H^{\prime}\right)$. We say $y<_{x} z$ if scanning the vertices of $H^{\prime}$ in clockwise direction along the circle starting from $x$, vertex $y$ is encountered before $z$. Let $x \in V\left(Q_{i}\right)$. Vertex $y$ is called the farthest neighbor of $x$ before $z$ if $y \in N_{H^{\prime}}(x), y \in V\left(Q_{i}\right) \cup V\left(Q_{i+1}\right) \cup V\left(Q_{i+2}\right), y<_{x} z$, and for every other $w \in N_{H^{\prime}}(x)$ either $z<_{x} w$ or $w \notin V\left(Q_{i}\right) \cup V\left(Q_{i+1}\right) \cup V\left(Q_{i+2}\right)$ or both.

Claim 4.2. There always exists a vertex which is the farthest neighbor of $x$ before $z$, unless $(x, z) \in$ $E\left(H^{\prime}\right)$ and $z \in V\left(Q_{i}\right) \cup V\left(Q_{i+1}\right) \cup V\left(Q_{i+2}\right)$.

\footnotetext{
(i) throughout this proof expressions involving subscripts of $u, P, Q$, and $Q^{\prime}$ are to be taken modulo $p$. Every such expression should be evaluated to a value in $\{0, \ldots, p-1\}$. For example, consider a vertex $u_{i}$, where $i<p$ Then, $p+i=i$.
} 
Let $\{A, B\}$ be the bipartition of the bipartite graph $H^{\prime}$. We categorize the edges of $H^{\prime}$ as follows: an edge $(x, y) \in E\left(H^{\prime}\right)$ is called an $l$-edge, if $l=\min \left(\operatorname{clock} \_d i s t(x, y), \operatorname{clock} \_d i s t(y, x)\right)$.

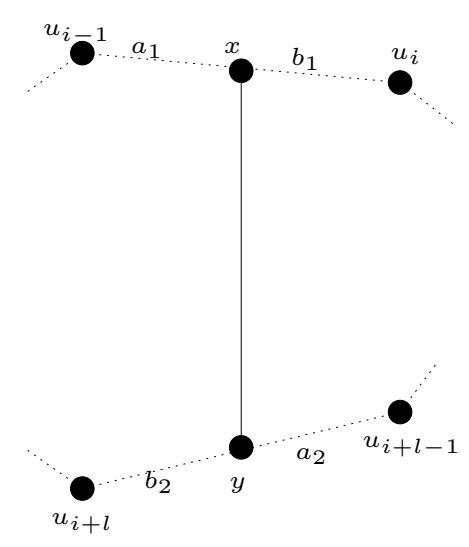

Fig. 1: $x \in V\left(Q_{i}\right), y \in V\left(Q_{i+l}\right)$ and let $(x, y) \in E\left(H^{\prime}\right)$ be an $l$-edge, where $l>2$. The dotted line between $u_{i-1}$ and $u_{i}$ indicate the path $Q_{i}$. Similarly, the dotted line between $u_{i+l-1}$ and $u_{i+l}$ indicate the path $Q_{i+l}$.

Claim 4.3. $H^{\prime}$ cannot have an $l$-edge, where $l>2$.

Proof: Suppose $H^{\prime}$ has an $l$-edge, where $l>2$, between $x \in Q_{i}$ and $y \in Q_{i+l}$ (see Fig. 1). Let $a_{1}=\left\|u_{i-1} Q_{i}^{\prime} x\right\|, b_{1}=\left\|x Q_{i}^{\prime} u_{i}\right\|, a_{2}=\left\|u_{i+l-1} Q_{i+l}^{\prime} y\right\|$ and $b_{2}=\left\|y Q_{i+l}^{\prime} u_{i+l}\right\|$. We consider the following two cases:

Case 1: $l$ is even

In this case $u_{i-1}$ and $u_{i+l-1}$ will be on the same side of the bipartite graph $H^{\prime}$. Without loss of generality, let $u_{i-1}, u_{i+l-1} \in A$. Then, $u_{i}, u_{i+l} \in B$. We know that, for every $w_{1}, w_{2} \in V\left(H^{\prime[m]}\right)$ with $w_{1} \in A$ and $w_{2} \in B$, if $\left(w_{1}, w_{2}\right) \notin E\left(H^{\prime[m]}\right)$ then $d_{H^{\prime}}\left(w_{1}, w_{2}\right) \geq m+2$ (recalling $m$ and $d_{H^{\prime}}\left(w_{1}, w_{2}\right)$ are odd integers). Therefore, we have $a_{1}+1+b_{2} \geq d_{H^{\prime}}\left(u_{i-1}, u_{i+l}\right) \geq m+2$. Similarly, $b_{1}+1+a_{2} \geq$ $d_{H^{\prime}}\left(u_{i}, u_{i+l-1}\right) \geq m+2$. Summing up the two inequalities we get, $\left(a_{1}+b_{1}\right)+\left(a_{2}+b_{2}\right) \geq 2 m+2$. This implies that either $\left\|Q_{i}^{\prime}\right\|$ or $\left\|Q_{i+l}^{\prime}\right\|$ is greater than $m$ which is a contradiction.

Case 2 : $l$ is odd

(proof is similar to the above case and hence omitted).

Hence we prove the claim.

We find a cycle $C^{\prime}=z_{0} z_{1} \ldots z_{q} z_{0}$ in $H^{\prime}$ using Algorithm $3.1^{(\text {i) }}$. Please read the algorithm before proceeding further. .

\footnotetext{
(i) throughout this proof expressions involving subscripts of $z$ are to be taken modulo $q+1$. Every such expression should be evaluated to a value in $\{0, \ldots, q\}$. For example, consider a vertex $z_{a}$, where $a<q+1$. Then, $q+1+a=a$.
} 


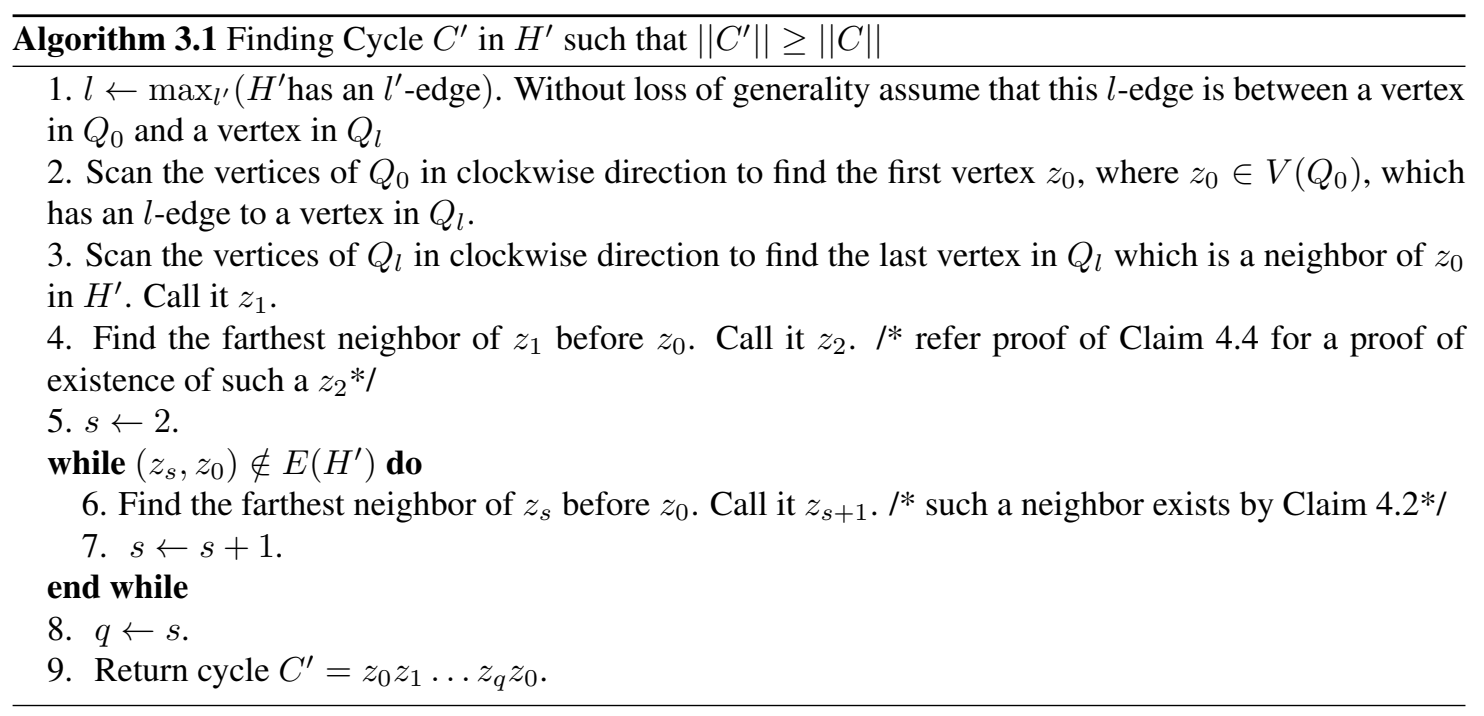

Claim 4.4. There always exists a farthest neighbor of $z_{1}$ before $z_{0}$.

Proof: Note that $z_{0} \in Q_{0}$ and $z_{1} \in Q_{l}$, where $l \leq 2$ (by Claim 4.3). Recalling that $\|C\|=p \geq 6$, we have $z_{0} \notin V\left(Q_{l}\right) \cup V\left(Q_{l+1}\right) \cup V\left(Q_{l+2}\right)$. Hence by Claim 4.2 , the claim is true.

Claim 4.5. The while loop in Algorithm 3.1 terminates after a finite number of iterations.

Proof: From Claim 4.1, we know that each vertex has an edge (in $H^{\prime}$ ) with both its left neighbor and right neighbor in the circular arrangement. Each time when Step 6 of Algorithm 3.1 is executed, a vertex $z_{s+1}$ is chosen such that $z_{s+1}$ is the farthest neighbor of $z_{s}$ before $z_{0}$. Since $H^{\prime}$ is a finite graph, there will be a point of time in the execution of the algorithm when in Step 6 it picks a $z_{s+1}$ such that $\left(z_{s+1}, z_{0}\right) \in$ $E\left(H^{\prime}\right)$.

From Claim 4.5, we can infer that $C^{\prime}$ is a cycle.

Claim 4.6. $C^{\prime}$ is an induced cycle in $H^{\prime}$.

Proof: Suppose $C^{\prime}$ is not an induced cycle. Then there exists a chord $\left(z_{a}, z_{b}\right)$ in $C^{\prime}$. Since $\left(z_{a}, z_{b}\right)$ is a chord, we have $b \neq a-1$ or $b \neq a+1$. Let $l=\max _{l^{\prime}}\left(H^{\prime}\right.$ has an $l^{\prime}$-edge). Let $z_{a} \in V\left(Q_{i}\right)$, $z_{b} \in V\left(Q_{j}\right)$. We know that $\min \left(\operatorname{clock} \_\operatorname{dist}\left(z_{a}, z_{b}\right)\right.$, clock_dist $\left.\left(z_{b}, z_{a}\right)\right) \leq l$. Without loss of generality, assume clock_dist $\left(z_{a}, z_{b}\right) \leq l \leq 2$ (from Claim 4.3 . That is, $j-i \leq l \leq 2$ and $\left(z_{a}, z_{b}\right)$ is a $(j-i)$-edge. If $z_{a}=z_{0}$, then $z_{b} \neq z_{1}$ and the algorithm exits from the while loop, when $q=b$, thus returning a cycle $z_{0} \ldots z_{b} z_{0}$. But in such a cycle $\left(z_{b}, z_{0}\right)$ is not a chord. Therefore, $z_{a} \neq z_{0}$. Similarly, $z_{b} \neq z_{0}$. We know that $z_{a+1} \neq z_{b}, z_{a+1}<_{z_{a}} z_{b}$, and $z_{a+1} \in V\left(Q_{i}\right) \cup V\left(Q_{i+1}\right) \cup V\left(Q_{i+2}\right)$. Since $j-i \leq 2$, $z_{b} \in V\left(Q_{i}\right) \cup V\left(Q_{i+1}\right) \cup V\left(Q_{i+2}\right)$. If $z_{b}<_{z_{a}} z_{0}$, then it contradicts the fact that $z_{a+1}$ is the farthest neighbor of $z_{a}$ before $z_{0}$. Therefore, $z_{0}<_{z_{a}} z_{b}$. Then, either $z_{b}=z_{1}$ or $z_{1}<_{z_{a}} z_{b}$. Recall that $l=\max _{l^{\prime}}\left(H^{\prime}\right.$ has an $l^{\prime}$-edge $)$, and $\left(z_{0}, z_{1}\right)$ is an $l$-edge with $z_{0} \in V\left(Q_{0}\right)$ and $z_{1} \in V\left(Q_{l}\right)$. Since (i) $\left(z_{a}, z_{b}\right)$ is a $(j-i)$-edge, where $j-i \leq l$, (ii) $z_{0}<_{z_{a}} z_{b}$, and (iii) $z_{b}=z_{1}$ or $z_{1}<_{z_{a}} z_{b}$, we have $l \geq j-i=$ clock_dist $\left(z_{a}, z_{b}\right) \geq \operatorname{clock\_ dist}\left(z_{0}, z_{b}\right) \geq \operatorname{clock} \_\operatorname{dist}\left(z_{0}, z_{1}\right)=l$. Hence, $j-i=l$ and 


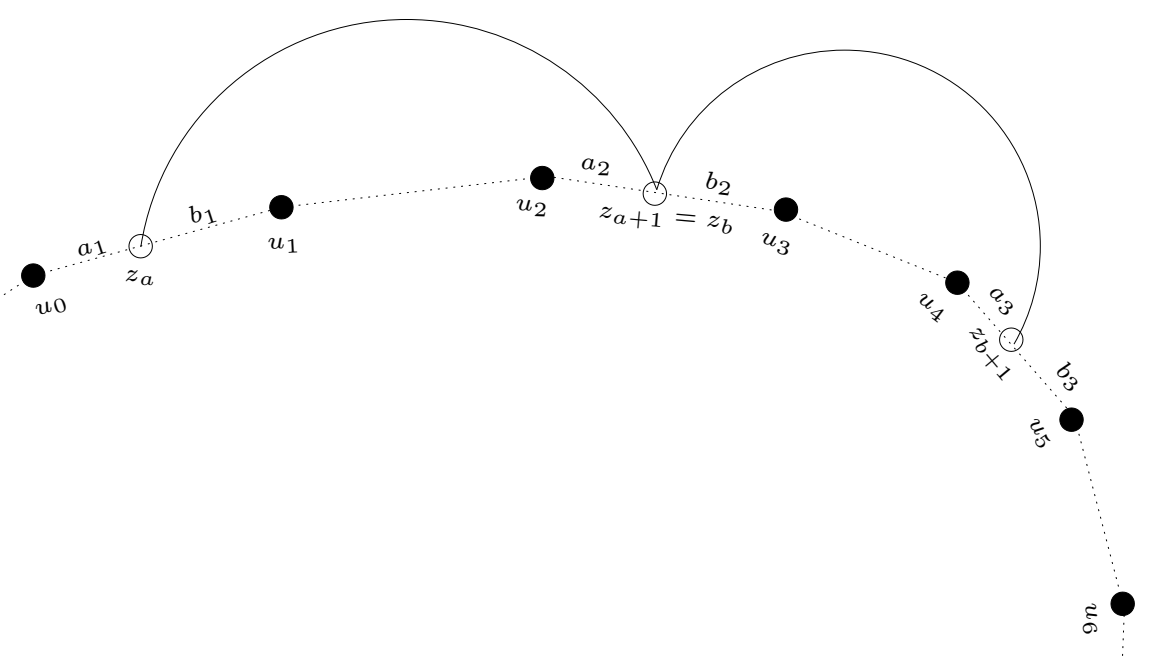

Fig. 2: Figure illustrates the case when path $P$ defined in Claim 4.8 is a trivial path. The dotted lines between each $u_{i-1}$ and $u_{i}$ indicate the path $Q_{i}^{\prime}$. Each continuous arc corresponds to an edge in the cycle $C^{\prime}=z_{0} \ldots z_{q} z_{0}$.

$\left(z_{a}, z_{b}\right)$ is an $l$-edge. We know that $\left(z_{0}, z_{1}\right)$ is also an $l$-edge with $z_{0} \in V\left(Q_{0}\right)$ and $z_{1} \in V\left(Q_{l}\right)$. Since $z_{0}<_{z_{a}} z_{b}$ and $z_{b}=z_{1}$ or $z_{1}<_{z_{0}} z_{b}$, we get $z_{a} \in V\left(Q_{0}\right)$ and $z_{b} \in V\left(Q_{l}\right)$. From Step 2 of the algorithm we know that $z_{0}$ is the first vertex (in a clockwise scan) in $Q_{0}$ which has an $l$-edge to a vertex in $Q_{l}$. This implies that, since $z_{0}<_{z_{a}} z_{b}, z_{a}=z_{0}$ which is a contradiction. Hence we prove the claim.

What is left now is to show that $q+1 \geq p$, i.e., $\left\|C^{\prime}\right\| \geq\|C\|$, where $C^{\prime}=z_{0} \ldots z_{q} z_{0}$ and $C=$ $u_{0} \ldots u_{p-1} u_{0}$. In order to show this, we state and prove the following claims.

Claim 4.7. For every $j \in\{0, \ldots, p-1\},\left(V\left(Q_{j}\right) \cup V\left(Q_{j+1}\right)\right) \cap V\left(C^{\prime}\right) \neq \emptyset$.

Proof: Suppose the claim is not true. Find the minimum $j$ that violates the claim. Clearly, $j \neq 0$ as $z_{0} \in V\left(Q_{0}\right)$. We claim that $z_{q} \in V\left(Q_{j-1}\right)$. Suppose $z_{q} \notin V\left(Q_{j-1}\right)$. Let $a=\max \left\{i \mid z_{i} \in\right.$ $\left.V\left(Q_{j-1}\right)\right\}$ (note that, since $j \neq 0$, by the minimality of $j,\left(V\left(Q_{j-1}\right) \cup V\left(Q_{j}\right)\right) \cap V\left(C^{\prime}\right) \neq \emptyset$ and therefore $\left.V\left(Q_{j-1}\right) \cap V\left(C^{\prime}\right) \neq \emptyset\right)$. Since $z_{a} \neq z_{q}$, by the maximality of $a$, we have $z_{a+1} \notin V\left(Q_{j-1}\right)$. From our assumption, $\left(V\left(Q_{j}\right) \cup V\left(Q_{j+1}\right)\right) \cap V\left(C^{\prime}\right)=\emptyset$ and therefore $z_{a+1} \notin V\left(Q_{j-1}\right) \cup V\left(Q_{j}\right) \cup V\left(Q_{j+1}\right)$. Thus $z_{a} \neq z_{q}$ and $z_{a+1}$ is not the farthest neighbor of $z_{a}$ before $z_{0}$. This is a contradiction to the way $z_{a+1}$ is chosen by Algorithm 3.1. Hence, $z_{q} \in V\left(Q_{j-1}\right)$. We know that $\left(z_{q}, z_{0}\right) \in E\left(H^{\prime}\right)$ with $z_{q} \in V\left(Q_{j-1}\right)$ and $z_{0} \in V\left(Q_{0}\right)$. Since $l=\max _{l^{\prime}}\left(H^{\prime}\right.$ has an $l^{\prime}$-edge $)$, we have min $\left(\operatorname{clock}{ }_{-} \operatorname{dist}\left(z_{q}, z_{0}\right)\right.$, clock_dist $\left.\left(z_{0}, z_{q}\right)\right) \leq l$. That is, $j \geq p+1-l$ or $j \leq 1+l$. As $l \leq 2$ (by Claim 4.3, we have $j=p-1$ or $j \leq 1+l$. Since $z_{0} \in V\left(Q_{0}\right),\left(V\left(Q_{p-1}\right) \cup V\left(Q_{0}\right)\right) \cap V\left(C^{\prime}\right) \neq \emptyset$ and hence $j \neq p-1$. Therefore, $j \leq 1+l$. Since $z_{0} \in V\left(Q_{0}\right)$ and $z_{1} \in V\left(Q_{l}\right)$ (recall $\left.l \leq 2\right)$, we get $j=1+l$. We know that, for every $z_{a}, z_{b} \in V\left(C^{\prime}\right)$, if $a<b$ then $z_{a}<_{z_{0}} z_{b}$. Therefore, $z_{1}<_{z_{0}} z_{q}$. We have $z_{1} \in V\left(Q_{l}\right)$. Since $j=1+l$, we also have $z_{q} \in V\left(Q_{l}\right)$. Thus, we have $z_{1}, z_{q} \in V\left(Q_{l}\right)$ and $z_{1}<_{z_{0}} z_{q}$. But this contradicts the fact that $z_{1}$ is the last vertex in $Q_{l}$ encountered in a clockwise scan that has $z_{0}$ as its neighbor.

Claim 4.8. Let $\left(z_{a}, z_{a+1}\right),\left(z_{b}, z_{b+1}\right) \in E\left(C^{\prime}\right)$ be two 2-edges, where $a<b$. Let $P, P^{\prime}$ denote the clockwise $z_{a+1}-z_{b}, z_{b+1}-z_{a}$ paths respectively in $C^{\prime}$. Both $P$ and $P^{\prime}$ contain at least one 0 -edge. 

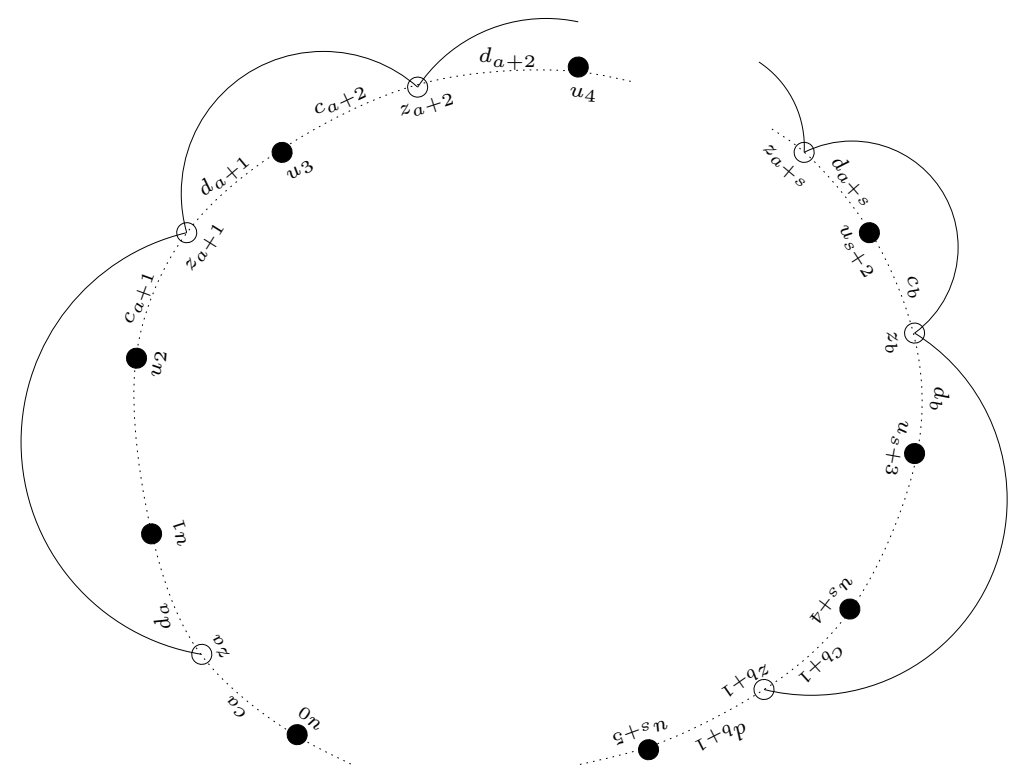

Fig. 3: Figure illustrates the case when path $P$ defined in Claim 4.8 is $P=z_{a+1} z_{a+2} \ldots z_{a+1+s}$, where $s \geq 1$ and $z_{a+1+s}=z_{b}$. The dotted lines between each $u_{i-1}$ and $u_{i}$ indicate the path $Q_{i}^{\prime}$. Each continuous arc corresponds to an edge in the cycle $C^{\prime}=z_{0} \ldots z_{q} z_{0}$.

Proof: Consider the path $P$ (proof is similar in the case of path $P^{\prime}$ ). Path $P$ is a non-trivial path only if $z_{a+1} \neq z_{b}$. Suppose $z_{a+1}=z_{b}$ (see Fig. 2). Let $z_{a} \in V\left(Q_{f}\right)$. For the sake of ease of notation, assume $f=1$ (the same proof works for any value of $f$ ). Let $a_{1}=\left\|u_{0} Q_{1}^{\prime} z_{a}\right\|, b_{1}=\left\|z_{a} Q_{1}^{\prime} u_{1}\right\|$, $a_{2}=\left\|u_{2} Q_{3}^{\prime} z_{b}\right\|, b_{2}=\left\|z_{b} Q_{3}^{\prime} u_{3}\right\|, a_{3}=\left\|u_{4} Q_{5}^{\prime} z_{b+1}\right\|$, and $b_{3}=\left\|z_{b+1} Q_{5}^{\prime} u_{5}\right\|$. We know that, for every $w_{1}, w_{2} \in V\left(H^{\prime[m]}\right)$ with $w_{1} \in A$ and $w_{2} \in B$, if $\left(w_{1}, w_{2}\right) \notin E\left(H^{\prime[m]}\right)$ then $d_{H^{\prime}}\left(w_{1}, w_{2}\right) \geq m+2$. Since $\left(u_{0}, u_{3}\right) \notin E\left(H^{\prime[m]}\right),\left(u_{1}, u_{4}\right) \notin E\left(H^{\prime[m]}\right)$ and $\left(u_{2}, u_{5}\right) \notin E\left(H^{\prime[m]}\right)$, we have $a_{1}+b_{2} \geq m+1$, $b_{1}+a_{3} \geq m$, and $a_{2}+b_{3} \geq m+1$. Adding the three inequalities and by applying an easy averaging argument we can infer that either $a_{1}+b_{1}=\left\|Q_{1}\right\|>m, a_{2}+b_{2}=\left\|Q_{3}\right\|>m$, or $a_{3}+b_{3}=\left\|Q_{5}\right\|>m$ which is a contradiction. Therefore $P$ is a non-trivial path i.e., $z_{a+1} \neq z_{b}$. Assume $P$ does not contain any 0 -edge. Let $P=z_{a+1} z_{a+2} \ldots z_{a+1+s}$, where $s \geq 1, a+1+s=b$, and $\left(z_{a+1}, z_{a+2}\right) \ldots\left(z_{a+s}, z_{a+1+s}\right)$ are 1-edges (see Fig. 3 . Since $\left(u_{0}, u_{3}\right) \notin E\left(H^{\prime[m]}\right),\left(u_{1}, u_{4}\right) \notin E\left(H^{\prime[m]}\right)$, we have $c_{a}+d_{a+1} \geq m+1$ and $d_{a}+d_{a+2} \geq m$ (please refer Fig. 3 for knowing what $c_{a}, d_{a}, \ldots, c_{b+1}, d_{b+1}$ are). Summing up the two inequalities, we get $d_{a+1}+d_{a+2} \geq 2 m+1-\left(c_{a}+d_{a}\right)$. We know that, for each $i \in\{0, \ldots p-1\}$, $\left\|Q_{i}^{\prime}\right\| \leq m$. Therefore, we have $c_{a}+d_{a} \leq m$. Hence, $d_{a+1}+d_{a+2} \geq m+1$. Since $\left(c_{a+1}+d_{a+1}\right)+$ $\left(c_{a+2}+d_{a+2}\right) \leq 2 m$, we get

$$
c_{a+1}+c_{a+2} \leq m-1
$$

Since $\left(u_{s+2}, u_{s+5}\right) \notin E\left(H^{\prime[m]}\right),\left(u_{s+1}, u_{s+4}\right) \notin E\left(H^{\prime[m]}\right)$, we have,

$$
\begin{aligned}
c_{b}+d_{b+1} & \geq m+1 \\
c_{a+s}+c_{b+1} & \geq m
\end{aligned}
$$


Summing up the two inequalities, we get

$$
c_{b}+c_{a+s} \geq 2 m+1-\left(c_{b+1}+d_{b+1}\right)
$$

Since $b=a+s+1$ and $c_{b+1}+d_{b+1} \leq m$, we get

$$
c_{a+s+1}+c_{a+s} \geq m+1
$$

Substituting for $s=1$ in Inequality 2, we get $c_{a+2}+c_{a+1} \geq m+1$. But this contradicts Inequality 1 . Hence $s>1$. Suppose $s=2$. Since $\left.\left(u_{2}, u_{5}\right) \notin E\left(H^{\prime[m]}\right)\right)$, we have $c_{a+1}+d_{a+3} \geq m$. Adding this with Inequality 2, we get $c_{a+1}+c_{a+2} \geq(2 m+1)-\left(c_{a+3}+d_{a+3}\right) \geq m+1$. But this contradicts Inequality 1 . Hence $s>2$. Since $\left.\left.\left(u_{s}, u_{s+3}\right) \notin E\left(H^{\prime[m]}\right)\right), \ldots,\left(u_{2}, u_{5}\right) \notin E\left(H^{\prime[m]}\right)\right)$, we have the following inequalities:-

$$
\begin{aligned}
c_{a+s-1}+d_{a+s+1} & \geq m \\
\vdots & \vdots \quad \vdots \\
c_{a+1}+d_{a+3} & \geq m
\end{aligned}
$$

Adding the above set of inequalities and applying the fact that $c_{i}+d_{i} \leq m, \forall i \in\{0, \ldots q\}$, we get $c_{a+1}+c_{a+2}+d_{a+s}+d_{a+s+1} \geq 2 m$. Adding this with Inequality 2 we get $c_{a+1}+c_{a+2} \geq(3 m+1)-$ $\left(c_{a+s+1}+d_{a+s+1}\right)-\left(c_{a+s}+d_{a+s}\right) \geq m+1$. But this contradicts Inequality 1. Hence we prove the claim.

Claim 4.9. For every $j, j^{\prime} \in\{0, \ldots, p-1\}$, where $j<j^{\prime}$ and $\left(V\left(Q_{j}\right) \cup V\left(Q_{j^{\prime}}\right)\right) \cap V\left(C^{\prime}\right)=\emptyset$, there exist $i, i^{\prime} \in\{0, \ldots, p-1\}$, where only $i$ satisfies $j<i<j^{\prime}$, such that $\left|V\left(Q_{i}\right) \cap V\left(C^{\prime}\right)\right| \geq 2$ and $\left|V\left(Q_{i^{\prime}}\right) \cap V\left(C^{\prime}\right)\right| \geq 2$.

Proof: By Claim 4.7, (i) $j^{\prime} \neq j+1$ or $j^{\prime} \neq j-1$, and (ii) there exist $r, r^{\prime} \in\{0, \ldots, q\}$ such that $\left(z_{r}, z_{r+1}\right)$ is a 2-edge with its endpoints on $Q_{j-1}$ and $Q_{j+1}$ and $\left(z_{r^{\prime}}, z_{r^{\prime}+1}\right)$ is a 2-edge with its endpoints on $Q_{j^{\prime}-1}$ and $Q_{j^{\prime}+1}$. By Claim 4.8, we know that if $P, P^{\prime}$ denote the clockwise $z_{r+1}-z_{r^{\prime}}, z_{r^{\prime}+1}-z_{r}$ paths respectively in $C^{\prime}$, then both $P$ and $P^{\prime}$ contains at least one 0 -edge. This proves the claim.

In order to show that the size of cycle $C^{\prime}\left(=z_{0} \ldots z_{q} z_{0}\right)$ is at least $p$, we consider the following three cases:-

Case $\left|\left\{Q_{j} \in\left\{Q_{0} \ldots Q_{p-1}\right\} \mid V\left(Q_{j}\right) \cap V\left(C^{\prime}\right)=\emptyset\right\}\right|=0$ : In this case, for every $j \in\{0, \ldots p-1\}, Q_{j}$ contributes to $V\left(C^{\prime}\right)$ and therefore $\left\|C^{\prime}\right\| \geq p=\|C\|$.

Case $\left|\left\{Q_{j} \in\left\{Q_{0} \ldots Q_{p-1}\right\} \mid V\left(Q_{j}\right) \cap V\left(C^{\prime}\right)=\emptyset\right\}\right|=1$ : Let $Q_{j}$ be that only path (among $Q_{0} \ldots Q_{p-1}$ ) that does not contribute to $V\left(C^{\prime}\right)$. Then we claim that there exists a $Q_{j^{\prime}}$, where $j^{\prime} \neq j$, such that $V\left(C^{\prime}\right) \cap V\left(Q_{j^{\prime}}\right) \geq 2$. Suppose the claim is not true then it is easy to see that $\left\|C^{\prime}\right\|=p-1$ which is an odd number thus contradicting the bipartitedness of $H^{\prime}$. Hence the claim is true. Now, by applying the claim it is easy to see that $\left\|C^{\prime}\right\|=\sum_{j}\left|V\left(C^{\prime}\right) \cap V\left(Q_{j}\right)\right| \geq p=\|C\|$.

Case $\left|\left\{Q_{j} \in\left\{Q_{0} \ldots Q_{p-1}\right\} \mid V\left(Q_{j}\right) \cap V\left(C^{\prime}\right)=\emptyset\right\}\right|>1$ : Scan vertices of $H^{\prime}$ starting from any vertex in clockwise direction. Claim 4.9 ensures that between every $Q_{j}$ and $Q_{j^{\prime}}$, which do not contribute to $V\left(C^{\prime}\right)$, encountered there exists a $Q_{i}$ which compensates by contributing at least two vertices to $V\left(C^{\prime}\right)$. Therefore, $\left\|C^{\prime}\right\| \geq p=\|C\|$. 


\section{Discussion}

An interesting open question that naturally follows from our result is the following: given a graph $G$ and positive integers $k, m$ where $k \geq 4$, if $G^{[m]}$ is $k$-chordal, then is $G^{[m+2]}$ also $k$-chordal? As mentioned earlier, Brandstädt et al. in [3] showed a similar result in the context of ordinary graph powering. They showed that, for every graph $G$, if $G^{m}$ is $k$-chordal, then so is $G^{m+2}$, where $k, m$ are positive integers with $k \geq 3$. A straightforward extension of their proof technique doesn't seem to work in our context due to the bipartite nature of the powering that we consider.

\section{References}

[1] R. Balakrishnan and P. Paulraja. Powers of chordal graphs. J. Aust. Math. Soc. Ser. A, 35:211-217, 1983.

[2] H. L. Bodlaender and D. M. Thilikos. Treewidth for graphs with small chordality. Discrete Applied Mathematics, 79:45-61, 1997.

[3] Andreas Brandstädt, Van Bang Le, and Thomas Szymczak. Duchet-type theorems for powers of hhd-free graphs. Discrete Mathematics, 177(1-3):9-16, 1997.

[4] L. Sunil Chandran, Mathew C. Francis, and Rogers Mathew. Chordal bipartite graphs with high boxicity. Graphs and Combinatorics, 27(3):353-362, 2011.

[5] L. Sunil Chandran and L. Shankar Ram. On the number of minimum cuts in a graph. In Proceedings of the 8th International computing and combinatorics conference, LNCS 2387, pages 220-230, 2002.

[6] L. Sunil Chandran, C.R. Subramanian, and Vadin V. Lozin. Graphs of low chordality. To appear in Dicrete Mathematics and Theoretical Computer Science, 2005.

[7] Gerard J. Chang and George L. Nemhauser. The $k$-domination and $k$-stability problems on sun-free chordal graphs. SIAM Journal on Algebraic and Discrete Methods, 5(3):332-345, 1984.

[8] Yon Dourisboure. Compact routing schemes for generalised chordal graphs. Journal of Graph Algorithms and Applications, 9:277-297, 2005.

[9] Feodor F. Dragan. Estimating all pairs shortest paths in restricted graph families: a unified approach. Journal of Algorithms, 57(1):1 - 21, 2005.

[10] P. Duchet. Classical perfect graphs. Ann. Discrete Math., 21:67-96, 1984.

[11] Carsten Flotow. On powers of m-trapezoid graphs. Discrete Appl. Math., 63(2):187-192, 1995.

[12] Carsten Flotow. On powers of circular arc graphs and proper circular arc graphs. Discrete Appl. Math., 69(3):199-207, 1996.

[13] A Lubiw. $\gamma$-free matrices. Master's thesis, Dept. of combinatorics and Optimization, University of Waterloo, 1982.

[14] A. Raychaudhuri. On powers of interval and unit interval graphs. Congr.Numerantium, 59:235-242, 1987. 
[15] A. Raychaudhuri. On powers of strongly chordal and circular graphs. Ars Combinatoria, 34:147160, 1992.

[16] Jeremy P. Spinrad. Finding large holes. Information Processing Letters, 39(4):227 - 229, 1991. 